\title{
Os impactos das barragens hidrelétricas na pesca artesanal amazônica sob o olhar dos sistemas socioecológicos
}

\begin{abstract}
A expansão de hidrelétricas na Amazônia tem sido a principal ameaça a sustentabilidade da pesca, mesmo com a implementação de medidas mitigadoras de seus impactos. Considerando que a compensação dos impactos se baseie nos estudos de impactos ambientais, para contribuir com o tema foi desenvolvida uma ferramenta de análise da qualidade de tais documentos a partir do arcabouço dos Sistemas Socioecológicos (SSE). O SSE foi aplicado aos casos de Estreito e Santo Antônio, demonstrando ser de fácil manuseio e potencialmente útil para tomadas de decisão. A análise dos casos evidenciou lacunas de informações importantes sobre o sistema pesqueiro, demonstrando serem insuficientes para a avaliação sistêmica dos impactos e, consequentemente, para a proposição de medidas mitigadoras. Assim, o presente estudo recomenda a aplicação dessa ferramenta para aperfeiçoar os estudos ambientais com vistas a sustentabilidade da atividade. Para tal, apresenta-se um guia de cinco passos que orienta a aplicação do arcabouço.
\end{abstract}

Palavras-chave: Abordagem interdisciplinar; Hidrelétricas; Licenciamento ambiental; Sistemas socioecológicos; Pesca artesanal.

\section{The impacts of hydroelectric dams on amazonian artisanal fisheries from the perspective of socio-ecological systems}

\begin{abstract}
The implementation of dams in the Amazon threatens fisheries, which can affect the food security of thousands of socially vulnerable families. Though mandatory, compensation for the dam's impacts on artisanal fishery systems has not achieved its expected outcomes. The environmental impact assessments have been insufficient to inform a systemic evaluation of these impacts. This study proposes a tool for evaluating dam's environmental impacts on Amazonian artisanal fisheries through an adaptation of the social-ecological systems (SES) framework. This framework was applied to test the impacts of the Estreito and Santo Antônio dams, proving useful, easy to handle, and visually appealing. The tool enabled the identification of missing or incomplete information that should be included or more deeply considered in environmental impact studies. Information gaps were evidenced for both hydroelectric, which may compromise the sustainable management of the activity. A five-step guide to implementing this tool is presented in the final section.
\end{abstract}

Keywords: Interdisciplinary approach; Hydroelectric dams; Environmental licensing; Social-ecological systems; Artisanal fishing.

Topic: Uso Sustentável da Biodiversidade

Reviewed anonymously in the process of blind peer
Received: 02/10/2020

Approved: 22/11/2020
Dernival Venâncio Ramos Júnior (iD) Universidade Federal do Tocantins, Brasil http://lattes.cnpq.br/9941464654933458 http://orcid.org/0000-0001-5092-1199 dernivaljunior@gmail.com

Ellen Silvia Amaral Figueiredo (iD Universidade Federal do Tocantins, Brasil http://lattes.cnpq.br/8965277422189321 http://orcid.org/0000-0002-2474-8449 ellenamaral@hotmail.com

Carolina Rodrigues da Costa Doria (iD Universidade Federal de Rondônia, Brasil http://lattes.cnpq.br/6716883529427154 http://orcid.org/0000-0003-1638-0063 carolinarcdoria@unir.br

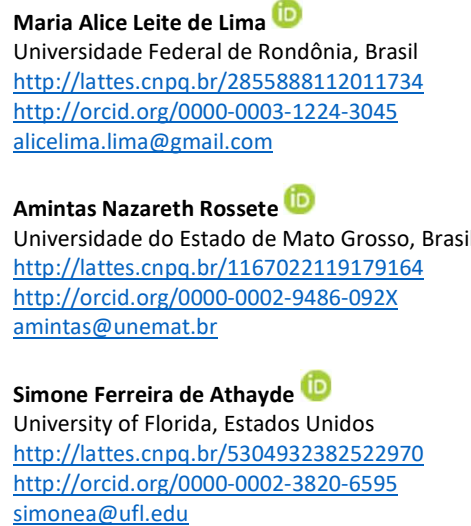

Elineide Eugênio Marques (iD Universidade Federal do Tocantins, Brasil http://lattes.cnpq.br/9875056556045599 http://orcid.org/0000-0003-0223-6853 emarques@mail.uft.edu.br

Neiva Cristina de Araújo

Universidade Federal de Rondônia, Brasil http://lattes.cnpq.br/7300866906734717 http://orcid.org/0000-0003-3252-4514 araujo.nc@gmail.com

Walterlina Barboza Brasil (iD

Universidade Federal de Rondônia, Brasi http://lattes.cnpq.br/3555085551615800 http://orcid.org/0000-0002-0905-1838 wal@unir.br
DOI: 10.6008/CBPC2179-6858.2020.006.0018
Referencing this:

RAMOS JÚNIOR, D. V.; FIGUEIREDO, E. S. A.; DORIA, C. R. C.; LIMA, M. A. L.; ROSSETE, A. N.; ATHAYDE, S. F.; MARQUES, E. E.; ARAÚJO, N. C.; BRASIL, W. B.. Os impactos das barragens hidrelétricas na pesca artesanal amazônica sob o olhar dos sistemas socioecológicos. Revista Ibero Americana de Ciências Ambientais, v.11, n.6, p.209-223, 2020. DOI: http://doi.org/10.6008/CBPC2179-6858.2020.006.0018 


\section{INTRODUÇÃO}

A eminente diminuição de recursos como as matas ciliares, a água e os peixes, desencadeada pelo modelo de desenvolvimento econômico vigente, é um dos principais problemas a serem enfrentados neste século (DUARTE et al., 2017; OSTROM, 2009; WINEMILLER et al., 2016). A conservação dos recursos pesqueiros, por exemplo, encontra-se ameaçada pelo aumento do consumo humano e pela alteração dos seus ambientes, seja através da poluição de rios e mares, das mudanças climáticas ou dos projetos de infraestrutura (AGOSTINHO et al., 2016; BHAUMIK et al., 2017; COOPER et al., 2016; LITTLE, 2002; OLIVEIRA et al., 2016; SANTOS et al., 2005; WINEMILLER et al., 2016).

A sustentabilidade da pesca de pequena escala na Amazônia tem sido desafiada pela implementação de grandes obras de desenvolvimento tais como portos, pontes, hidrovias e grandes empreendimentos hidrelétricos que impactam as bacias hidrográficas em toda a sua extensão (CASTRO, 2012; FEARNSIDE, 2014; MORAN, 2016). Dentre os grandes projetos, as barragens hidrelétricas são as que causam maior distúrbio no ambiente, pois são, como afirma Oliver-Smith (2010), obras totalizantes que afetam os sistemas socioecológicos como um todo, podendo levá-los à desarticulação e/ou colapso. Na Amazônia e em outras regiões tropicais, a construção de barragens hidrelétricas tem impactado a vida de milhares de famílias que dependem da pesca artesanal para sua sobrevivência (AGOSTINHO et al., 2004; DORIA et al., 2012). Neste sentido, a busca por ferramentas para o enfrentamento dos problemas em ambientes de hidrelétricas é urgente e será abordada no presente trabalho.

As ameaças à sustentabilidade dos recursos pesqueiros em ambientes transformados por hidrelétricas podem levar ao que Hardin descreveu como "tragédia dos comuns" (HARDIN, 1968). Sem a atuação efetiva do Estado ou da iniciativa privada, o futuro dos recursos pesqueiros sob o controle dos próprios usuários seria a depleção completa. Essa teoria partiu da preocupação do autor com o aumento exponencial da população humana em um cenário de recursos naturais finitos. A principal opositora da 'Tragédia' foi a economista Elinor Ostrom. Além de questionar a privatização ou a estatização como mecanismo eficaz para impedir a sobre-exploração dos recursos naturais, ela argumentou que os 'comuns', sob determinadas condições, poderiam sim ser capazes de contribuir para a conservação dos recursos naturais por meio da auto-organização. Para defender a Teoria do Uso Comum dos Recursos Naturais, Ostrom e seus colaboradores reuniram, ao longo de anos, evidências de milhares de casos de boa governança dos recursos naturais e propuseram uma lista de oito princípios presentes nos casos de sucesso observados. Por ter demonstrado que a participação dos usuários poderia levar a sustentabilidade, Ostrom foi agraciada com o prêmio Nobel de ciências econômicas em 2009, e nas últimas décadas, sua teoria tem influenciado a gestão dos recursos naturais no mundo todo (BASURTO et al., 2013; BERKES, 2011; BUSCHBACHER, 2014; OSTROM, 1990; 2009; OVIEDO et al., 2016; POTEETE et al., 2010).

Os princípios institucionais da teoria do uso comum dos recursos naturais (OSTROM, 1990), todavia, receberam diversas críticas por serem generalistas e negligenciarem fatores importantes que afetariam um determinado sistema socioambiental. A teoria institucionalista da qual parte Ostrom, leva a uma tentativa 
de universalização de certas regras que devem ser vistas com cuidado, pois as mesmas não apenas mudam socialmente, mas tem sentidos e significados culturalmente distintos nos diversos sistemas que se deseja estudar (WALL, 2015). Agrawal (2001) alerta que o boom de estudos sobre os Commons e a utilização de listas de condições propícias para a auto-organização dos usuários pode ser falho e custar caro. Para o autor, os casos estudados costumam ser de comunidades bem pequenas e muito diferentes entre si. Mesmo assim, ele reconhece a importância dessa perspectiva para orientar tomadas de decisão e propõe um maior rigor metodológico aos estudos, assim como um maior aprofundamento quanto ao recurso em foco.

Nesse sentido, rumo a um aprofundamento teórico metodológico, Ostrom e colaboradores (BERKES, 2011; OSTROM, 1990; POTEETE et al., 2010) passaram a desenvolver um quadro de atributos e variáveis para a busca de solução para os conflitos socioambientais, com abordagem interdisciplinar, que pudesse se adaptado a diversas realidades. Esse esforço culminou no arcabouço dos Sistemas Socioecológicos (SSE) (OSTROM, 2009). Para a autora, todo uso de um determinado recurso natural pode ser compreendido como um sistema socioecológico, que envolve pelo menos quatro subsistemas: Sistemas de Recurso - SR (ex. Sistema da pesca na Amazônia); Unidade de Recurso - UR (ex. espécies de peixes de importância comercial); Sistema de Governança - G (regras e sanções aplicadas ao sistema, governo e outras organizações envolvidas com o gerenciamento da pesca); e Atores - A (diferentes atores sociais envolvidos na atividade). Estes subsistemas, ao mesmo tempo em que interagem entre si, sofrem influência de questões políticas, sociais e econômicas, produzindo diferentes resultados (OSTROM, 2009).

O arcabouço de Sistemas Socioecológicos (SSE) foi desenvolvido para contribuir de forma prática com a busca por soluções aos problemas socioambientais de natureza complexa (OSTROM, 2009), como o caso estudado por este artigo. Um dos principais desafios enfrentados pelos idealizadores da ferramenta foi superar as barreiras disciplinares, integrando os saberes de várias disciplinas científicas que tradicionalmente trabalharam isoladas (OSTROM, 1990; 2009). Além disso, o arcabouço deveria ser aplicável a diferentes casos e sistemas, englobando paritariamente estudos para a compreensão das dimensões sociais e ambientais, para resolver questões socioambientais complexas. Para Basurto et al. (2013), as causas do sucesso ou insucesso da gestão de um determinado recurso natural dificilmente podem ser atribuídas a uma única causa, e por isso mesmo, a único campo disciplinar, como tradicionalmente se fez. Somente através de uma abordagem interdisciplinar é possível conhecer e lidar com os problemas de ordem complexa (OSTROM, 2009). A aplicação desse arcabouço permitiria uma análise abrangente de um determinado sistema e podendo contribuir com tomadas de decisão para a gestão sustentável do mesmo.

Diversos estudos (BASURTO et al., 2013; BINDER et al., 2013; COX, 2014; OVIEDO et al., 2016; TRIMBLE et al., 2015) aplicaram o arcabouço dos SSE para analisar diferentes recursos de uso comum e tem demonstrado sua utilidade para lidar com questões ambientais de natureza complexa. O crescente uso da ferramenta pode estar relacionado à importância do uso de arcabouços como ferramentas de pesquisa multi e/ou interdisciplinar e gestão socioambiental, uma vez que podem apoiar o entendimento do funcionamento dos sistemas, bem como projetar os impactos que determinado tipo de ação podem provocar no mesmo 
(BASURTO et al., 2013).

Considerando a complexidade do sistema pesqueiro amazônico (SSESPA) no contexto de grandes usinas hidrelétricas, o SSE pode ser uma ferramenta interessante para orientar tomadas de decisão em relação à gestão dos recursos pesqueiros. Isso porque o modelo vigente de gestão parece ineficiente e tem sido criticado por pesquisadores experientes no tema (AGOSTINHO et al., 2004; DORIA et al., 2017). De um modo geral, a gestão pesqueira no Brasil tem sido historicamente desafiadora. Sob a responsabilidade do Estado, o gerenciamento da pesca ainda se dá de forma centralizada e sofre com notável ausência de recursos humanos e financeiros, apesar de avanços no sentido de promover a participação dos usuários na tomada de decisão (KALIKOSKI et al., 2009; SANTOS et al., 2005). O desinteresse das autoridades políticas no setor se justifica pela ineficiência no monitoramento da atividade, que subestima sua real importância para a economia e a segurança alimentar de inúmeras famílias de baixa renda no Brasil (DORIA et al., 2017; OLIVEIRA et al., 2016).

Em reservatórios hidrelétricos na Amazônia, gerir a pesca parece ainda mais desafiador (AGOSTINHO et al., 2016). Nas últimas décadas, as ações tomadas para o manejo da pesca em reservatórios brasileiros se resumem a monitoramento, estocagem e construção de escadas de peixe (AGOSTINHO et al., 2004). E essas medidas não têm surtido o efeito esperado. Soma-se a isso a falta de interesse das próprias empresas detentoras da exploração da hidroeletricidade em resolver o problema. Sem a aplicação de medidas compensatórias ou mitigadoras adequadas para a sustentabilidade da pesca artesanal, os impactos das barragens se potencializam. A atual forma de gestão de áreas à montante e à jusante das usinas não permite uma análise abrangente dos impactos das mesmas, e negligencia os direitos dos pescadores durante o processo de licenciamento ambiental (DORIA et al., 2017).

Sánchez (2013) evidenciou a importância de realização de estudos retrospectivos que apresentem avaliações críticas de estudos de impacto ambiental, para o aperfeiçoamento dos processos de avaliação de impacto ambiental (AIA) e capacitação de profissionais atuantes no sistema de licenciamento ambiental, tanto para a elaboração de termos de referência, quando para a análise de estudos apresentados pelas empresas responsáveis pela construção das obras. A existência de um conjunto de critérios ou de diretrizes preestabelecidos para orientar o trabalho do analista é um fator facilitador, ajudando na redução da subjetividade da análise, podendo levar a resultados mais consistentes e reprodutíveis em outras situações.

Nesse contexto, o presente trabalho tem como objetivo o desenvolvimento de uma ferramenta de avaliação da qualidade dos estudos ambientais de hidrelétricas na Amazônia, de forma a orientar a tomada de decisão com vistas à sustentabilidade. Parte-se do pressuposto de que parte do problema da gestão da pesca artesanal em rios afetados por hidrelétricas na Amazônia esteja relacionada à falta de informação suficiente para dimensionar os impactos ambientais. Se o levantamento e análise dos impactos não se dão de forma sistêmica, as medidas compensatórias propostas tenderão a falhar, desconsiderando variáveis e processos chave, correndo inclusive o risco de agravar o problema. Para enfrentar este desafio, e considerando a necessidade de uma análise sistêmica dos impactos para a implementação adequada de 
medidas compensatórias, como prevê as resoluções CONAMA № 001-A (23 de janeiro de 1986) e CONAMA № 237 (19 de dezembro de 1997), este trabalho toma como ponto de partida o arcabouço dos sistemas socioecológicos de Ostrom, adaptado para a pesca de pequena escala por Basurto et al. (2013). Com base nestes autores, propõe-se uma ferramenta de avaliação da qualidade dos estudos de impacto ambiental sobre a pesca artesanal na Amazônia, servindo de apoio para orientar e avaliar os estudos de avaliação de impacto em processos de tomada de decisão. A aplicabilidade do novo arcabouço foi testada na avaliação dos estudos de impacto ambiental de duas hidrelétricas na Amazônia: Santo Antônio (RO) e Estreito (TO/MA), possibilitando a análise de seus conteúdos do ponto de vista sistêmico. Ao final do artigo, sugere-se um guia de cinco passos para facilitar a aplicação do arcabouço SSE em sistemas da pesca artesanal da Amazônia.

\section{MATERIAIS E MÉTODOS}

O ponto de partida para o desenvolvimento da ferramenta de avaliação proposta por este estudo foi a adequação que Basurto et al. (2013) fizeram do arcabouço original de Sistemas Socioecológicos (SSE) (OSTROM, 2009) para estudar a pesca de peixes bentônicos. Reconhecendo a pesca artesanal como um sistema socioecológico complexo, os autores fizeram uma ampla revisão sistemática e criaram uma lista de variáveis-chave para cada subsistema do SSE a ser analisado: Sistemas de Recursos (SR), Unidades de Recursos (UR), Atores (A) e Sistemas de Governança (G) (BASURTO et al., 2013). O estudo destacou a importância da hierarquização das variáveis, sendo de primeira a quinta ordem, considerando o nível de aprofundamento sobre determinado tema.

O primeiro passo para a adequação do arcabouço SSE para a pesca em ambiente tropical amazônico foi revisar as variáveis existentes no arcabouço de Basurto et al. (2013) e fazer os ajustes necessários para sua adequação ao contexto estudado. Isso foi feito por uma equipe com formação multidisciplinar e proposta de trabalho interdisciplinar de 9 pesquisadores das áreas de humanas e biofísicas, com experiência em pesca e peixes na Amazônia. Parte da equipe tem experiência de mais de 10 anos trabalhando em ambientes impactados por barragens, com domínio da natureza e conteúdo dos documentos em análise. Os subsistemas SR e UR foram analisados por pesquisadores das biofísicas e os subsistemas A e G por pesquisadores das humanas. As definições das variáveis foram traduzidas e ajustadas, quando necessário. As variáveis não pertinentes foram excluídas do arcabouço e as novas variáveis, consideradas relevantes para o estudo, foram inseridas (ver Anexo l). Após ampla discussão da equipe por meio de reuniões presenciais ou virtuais, o novo arcabouço foi consolidado por meio de um quadro. O resumo das definições das variáveis, os atributos que foram excluídos, inseridos ou redefinidos pelos autores, bem como a literatura que amparou essas modificações encontram-se no Anexo I.

Uma vez definidas as variáveis importantes para se medirem os impactos de hidrelétricas na pesca amazônica, duas Usinas Hidrelétricas de grande porte (UHE, potência de geração >30MW) localizadas nas bacias dos rios Tocantins e Madeira foram selecionadas para ilustrar a aplicação do arcabouço desenvolvido, e avaliar os atributos e variáveis adequados para avaliar a sustentabilidade da pesca na Amazônia. $O$ critério 
para a seleção dos empreendimentos foi o fato delas serem obras do Programa de Aceleração do Crescimento (PAC). As usinas são a UHE de Estreito, no Rio Tocantins e a UHE de Santo Antônio, no Rio Madeira. A UHE de Estreito está situada nos municípios de Estreito (MA) e de Aguiarnópolis (TO). A usina entrou em funcionamento no ano de 2011, com uma área alagada de $400 \mathrm{~km} 2$ e uma potência instalada para gerar 1.087 MW de energia (CESTE, 2018). A UHE de Santo Antônio, por sua vez, está localizada no município de Porto Velho (RO), com uma área alagada de $421 \mathrm{~km} 2$ e uma potência instalada de $2.424 \mathrm{MW}$. A usina entrou em funcionamento no ano de 2008. A escolha destes estudos de caso também levou em consideração a familiaridade dos autores com estas usinas, e por realizarem pesquisas com foco na temática de peixes e pesca artesanal em ambas as regiões.

Os documentos utilizados na aplicação do novo arcabouço foram os Estudos de Impacto Ambiental (EIAs), os Relatórios de Impacto Ambiental (RIMA) e os Projetos Básicos Ambientais (PBA) de Estreito e Santo Antônio. A concessão das licenças ambientais de hidrelétricas (prévia-LP, de instalação-LI e de operação-LO) está vinculada aos termos de referência e análise dos EIA, RIMA e PBAs, que estimam os impactos sofridos através de estudos técnicos e propõem medidas mitigadoras ou compensatórias dos impactos, sendo considerados instrumentos básicos para a gestão de reservatórios de hidrelétricas. Essas exigências constam na Política Nacional do Meio Ambiental de 1981 (LEI № 6.938), e nas resoluções CONAMA № 001-A (23 de janeiro de 1986) e № 237 (19 de dezembro de 1997).

Para a aplicação do arcabouço, a equipe se dividiu novamente ficando 4 pessoas responsáveis pela sistematização dos documentos das duas hidrelétricas. Foi feita uma varredura dos documentos para identificação das partes que tratavam da pesca e da ictiofauna, sua leitura e análise. A análise teve início com a sistematização das informações em duas tabelas para cada subsistema (SR, UR, A, G). A primeira tabela sintetizava as informações qualitativamente, sendo registrados o tipo de informação e sua localização (documento e página). A segunda tabela categorizava a variável analisada de acordo com a consistência da informação encontrada, podendo ser classificada como: Presente (P), com informação adequada ou suficiente para avaliar o impacto ambiental; Presente Parcialmente (PP), com informações relevantes, mas com necessidade de aprofundamento; Insuficiente (I), com informações superficiais e insuficientes para avaliar o impacto; e Ausente (A), quando não havia informação sobre aquela variável. Uma vez preenchidas as tabelas, elas foram revisadas de acordo com cada área de atuação da equipe. Esta categorização foi estabelecida a partir do consenso entre os pesquisadores sobre as características das variáveis e a redação do conteúdo inerente à informação necessária à caracterização, dentro do documento. Após esta etapa, foi feita uma análise de frequência nos resultados. Todo o processo de desenvolvimento e aplicação da ferramenta aconteceu entre os anos de 2015 e 2018. As discussões e o aprendizado mútuo gerado foram fundamentais para o bom andamento do trabalho. 


\section{RESULTADOS}

\section{Adaptando o quadro de atributos e variáveis dos Sistemas Socioecológicos ao contexto da pesca artesanal} na Amazônia

O arcabouço desenvolvido por Ostrom (2009) e adaptado por Basurto et al. (2013) pode ser de grande utilidade para se avaliar a qualidade dos instrumentos EIA, RIMA e PBAs que medem os impactos, assim como embasam medidas mitigatórias dos impactos causados pelas barragens nos peixes e na pesca artesanal. Abaixo fornecemos um resumo das modificações feitas no quadro de variáveis de Basurto et al. (2013), adequando o mesmo à realidade da pesca na Amazônia com relação à estrutura das variáveis mínimas para se avaliar a sustentabilidade de determinado Sistema (QUADRO 1).

De modo geral, foram inseridas 9 variáveis novas, sendo quatro delas de segunda ordem, uma de terceira ordem, e quatro de quarta ordem. Foram redefinidas três variáveis de segunda ordem, cinco de terceira ordem, uma de quarta ordem e uma de quinta ordem. Apenas cinco variáveis do quadro de Basurto et al. (2013) foram excluídas, sendo uma de segunda ordem e quatro de terceira ordem (QUADRO 1). Essas alterações foram feitas no sentido de aprofundamento sobre os aspectos que influenciam a pesca em ambientes transformados por barragens hidrelétricas.

\section{Modificações detalhadas por subsistemas}

Para o subsistema SR houve inserção das variáveis de segundo nível SR10 e SR11; e de quarto nível SR4.2.1 e SR4.2.2. Foram também redefinidas a variável de primeiro nível SR1 e as de segundo nível SR1.1 e SR3.1. Essas alterações foram feitas no sentido de adequar a descrição das mesmas ao sistema de pesca em ambientes barrados da Amazônia.

As variáveis de segundo e terceiro nível SR7 e SR7.1, por sua vez, foram excluídas por não se aplicarem ao sistema estudado. A definição de SR foi adequada ao contexto estudado.

Com relação ao subsistema UR, não houve inserção de variáveis novas. Foram redefinidas as variáveis de segundo nível UR1 e de terceiro nível UR 3.2. Essas alterações foram feitas no sentido da descrição do sistema. Três variáveis de terceiro nível UR6.2, UR7.1 e UR7.2 não se aplicam ao sistema estudado e foram excluídas. A definição de UR foi adaptada ao contexto estudado.

Quadro 1: Variáveis para a avaliação da sustentabilidade do sistema pesqueiro Amazônico em ambientes impactados por barragens.

\begin{tabular}{|l|l|l|l|}
\hline & Sistema de recurso (SR) & A8.1.1 & Captura específica (novo) \\
\hline SR1 & Setor & A8.2 & Relevância cultural \\
\hline SR1.1 & $\begin{array}{l}\text { Espécies de peixes capturados pelos pescadores artesanais nas } \\
\text { bacias dos rios Madeira e Tocantins }\end{array}$ & A9 & Tecnologias disponíveis \\
\hline SR2 & Clareza das fronteiras do sistema & A9.1 & Material de pesca próprio \\
\hline SR3 & Tamanho do sistema do recurso & A9.2 & Homogeneidade \\
\hline SR3.1 & Capacidade de suporte & & \\
\hline SR4 & Produtividade do sistema & & Governança \\
\hline SR4.1 & Status do estoque pesqueiro & GS1 & Área política \\
\hline SR4.2 & Fatores biofísicos & GS1.1 & Meio ambiente \\
\hline SR4.2.1 & Presença de corredeiras (novo) & GS 1.1.1 & Pescarias \\
\hline
\end{tabular}




\begin{tabular}{|c|c|c|c|}
\hline SR4.2.2 & Presença ou não de tributários (novo) & GS2 & Alcance geográfico \\
\hline SR6 & $\begin{array}{l}\text { Predictability of systems dynamics (previsibilidade da dinâmica } \\
\text { de sistema) }\end{array}$ & GS3 & População \\
\hline SR7 & Características de armazenamento (Excluído) & GS4 & Tipo de regime \\
\hline SR7.1 & Armazenamento em ambiente natural (Excluído) & GS4.1 & Democracia \\
\hline SR7.2 & $\begin{array}{l}\text { Storage in a human-designed facility (armazenamento facilitado } \\
\text { por humanos) }\end{array}$ & GS4.1.1 & Representação indireta (novo) \\
\hline SR8 & Connectivity (Conectividade) & GS4.2 & Autocracia \\
\hline SR9 & Location (Localização) & GS5 & Organizações \\
\hline SR10 & Landscape (Novo) & GS5.1 & Organizações governamentais \\
\hline \multirow[t]{3}{*}{ SR11 } & Hydrodynamic (Novo) & GS5.1.1 & Suporte de fiscalização \\
\hline & & GS5.1.2 & Suporte técnico e financeiro \\
\hline & Unidade de recurso (UR) & GS5.1.3 & Esforços de restauração \\
\hline UR1 & Mobilidade da unidade de recurso & GS5.2 & $\begin{array}{l}\text { Organizações não governamentais e } \\
\text { movimentos sociais }\end{array}$ \\
\hline UR2 & Taxa de crescimento e reposição (renovação) & GS5.2.1 & Desenvolvimento de capacidades \\
\hline UR3 & Interação entre as unidades de recurso & GS5.2.2 & Conexão \\
\hline UR3.1 & Reprodução & GS5.2.3 & Ponte \\
\hline UR3.2 & Settlement (estabelecimento) & GS5.2.3.1 & União \\
\hline UR4 & Valor econômico & GS5.2.3.2 & Cooperativas \\
\hline UR5 & Número de unidades & GS6 & Regras em uso \\
\hline UR6 & Características distintivas & GS6.1 & Regime de propriedade \\
\hline UR6.1 & $\begin{array}{l}\text { Hatchery (Adaptar: manutenção de áreas de reprodução [para } \\
\text { garantir o repovoamento] }\end{array}$ & GS6.1.1 & Acesso livre \\
\hline UR6.2 & $\begin{array}{l}\text { Selvagem (marcações que ligam UR a sua origem selvagem) } \\
\text { (Excluído) }\end{array}$ & GS6.1.2 & $\begin{array}{l}\text { Moratórias ou total de capturas } \\
\text { permitidas }\end{array}$ \\
\hline UR7 & Distribuição espacial e temporal & GS6.1.3 & Capturas compartilhadas \\
\hline UR7.1 & Distribuição não homogênea (Excluído) & GS6.1.4 & Privilégios de uso territorial \\
\hline \multirow[t]{3}{*}{ UR7.2 } & Distribuição aleatória (Excluído) & GS6.1.4.1 & River tracts (definição de territórios) \\
\hline & & GS6.1.4.2 & Áreas individuais de pesca \\
\hline & Atores (A) & GS6.1.4.3 & Direitos de uso comum do território \\
\hline A1 & $\begin{array}{l}\text { Number of relevant actors (pescadores comerciais, subsistência } \\
\text { e indígenas) }\end{array}$ & GS6.2 & Regras operacionais \\
\hline A1.1 & Exploradores dos recursos (pescadores/atravessadores (novo) & GS6.3 & Regras de uso coletivo \\
\hline $\mathrm{A} 2$ & Atributos socioeconômicos & GS6.4 & Regras constitucionais \\
\hline $\mathrm{A3}$ & Histórias ou experiências anteriores & GS7 & Normas e estratégias \\
\hline A3.1 & Crises/conflitos & GS8 & Estrutura de Rede \\
\hline A3.2 & Duração & GS8.1 & Horizontal \\
\hline A4 & Localização & GS8.2 & Vertical \\
\hline A5 & Liderança & GS9 & Monitoramento \\
\hline A6 & Capital social & GS9.1 & Social \\
\hline A6.1 & Confiança e reciprocidade & GS9.2 & Biofísico \\
\hline A7 & Conhecimento do SSC e modelos mentais & GS10.1 & Sanções graduais \\
\hline A7.1 & Mecanismo para compartilhar conhecimentos sobre pesca & GS10.2 & Sanções drásticas \\
\hline A8 & Importância do recurso & GS11 & $\begin{array}{l}\text { Estratégias } \quad \text { de } \\
\text { implementadas (novo) }\end{array}$ \\
\hline A8.1 & Relevância econômica do recurso & GS12 & $\begin{array}{l}\text { Estratégias de comunicação e } \\
\text { convencimento (novo) }\end{array}$ \\
\hline \multicolumn{4}{|c|}{ Ecossistemas relacionados (ECO) } \\
\hline & & & \\
\hline
\end{tabular}

Fonte: Adaptado de Basurto et al. (2013).

No que diz respeito aos atributos elencados por Basurto et al. (2013) para o subsistema G, inserimos duas variáveis de segundo nível G11 e G12, e uma variável de quarto nível G4.1.2. Foram também redefinidas as variáveis de quinto e quarto nível G6.1.4.1 e G1.1.1. Essas alterações se deram no sentido de aprimorar a descrição do sistema.

Por fim, para o subsistema A foram inseridas as variáveis de terceiro nível A1.1 e quarto nível A8.1.1. As variáveis de segundo nível A7, de terceiro nível A8.1 e A8.2 foram redefinidas no sentido de aprimoramento conceitual e não de descrição do sistema. 


\section{Aplicando o quadro de atributos e variáveis aos casos dos rios Madeira e Tocantins}

Nos últimos 40 anos, as bacias hidrográficas estudadas foram fortemente impactadas por uma série de obras de desenvolvimento. Como obras totalizantes (OLIVER-SMITH, 2010), as usinas são, potencialmente, as mais impactantes delas. São oito as usinas hidrelétricas construídas nas últimas duas décadas, sendo Serra da Mesa, Cana Brava, Lajeado, Peixe Angical, Estreito e São Salvador no rio Tocantins; e Santo Antônio e Jirau no rio Madeira. Das duas bacias estudadas, a do rio Tocantins foi a primeira a ser impactada pela construção de barragens. A hidrelétrica de Tucuruí foi finalizada no ano de 1982. Nas duas décadas seguintes, houve uma expansão da fronteira enérgica brasileira para a Amazônia com a construção de Serra da Mesa (1994) e Lajeado (2001). A partir de 2006, uma série de novas hidrelétricas foi projetada e construída: Jirau (2010) e Santo Antônio (2011) no rio Madeira, e Estreito (2010) e Marabá (em fase inicial de construção) no rio Tocantins. Essa nova política para o aproveitamento dos recursos hídricos amazônicos, mormente chamada de neoextrativista (CASTRO, 2012; SEVÁ FILHO, 2008), tem impactado profundamente os recursos pesqueiros (AGOSTINHO et al., 2004; FEARNSIDE, 2014). A maioria das hidrelétricas citadas foi construída após a obrigatoriedade dos estudos de impacto ambiental, determinada pela resolução no 001 do CONAMA (1986).

Um segundo momento do trabalho foi testar a adaptação do novo arcabouço SSE para o ambiente a ser afetado por barragens a partir dos documentos do EIA, RIMA e PBA de Estreito e Santo Antônio. Ao se analisar o conteúdo destes documentos, constatou-se que um pouco menos de $50 \%$ das variáveis buscadas se encontraram ausentes. Dentre as informações constantes nos documentos, cerca de $25 \%$ delas se enquadram nas categorias de superficiais ou insuficientes. Somente entre $10 \%$ e $15 \%$ das variáveis analisadas encontraram-se plenamente contempladas nos documentos requeridos para emissão de licenças ambientais. Os dados encontrados demonstram o quanto são defasados os documentos que deveriam conter a avaliação dos impactos socioecológicos deflagrados pelas barragens. Além disso, o resultado sugere também que a interdisciplinaridade e a integração de áreas relevantes do conhecimento não se deram de maneira efetiva.

Ao se observarem os documentos analisando os subsistemas em separado encontrou-se que o Sistema de Recurso (SR) e a Unidade de Recurso (UR) foram mais completos segundo o arcabouço do que Governança (G) e Atores (A). Isso corrobora com outros estudos que citam a importância de se conhecer mais sobre as pessoas e o modo de gestão do lugar (AGOSTINHO et al., 2007). Os documentos da hidrelétrica de Santo Antônio apresentaram-se mais completos do que os de Estreito, com relação a SR (GRÁFICO 1).

Os documentos EIA, de Estreito, e RIMA, de Santo Antônio, são mais omissos quanto ao SR. Com relação aos PBAs, o de Santo Antônio mostrou-se mais completo do que o de Estreito. Este foi o subsistema melhor analisado para o caso de Santo Antônio (GRÁFICO 1A). Quanto a UR, as variáveis pareceram, em um panorama geral, mais deficitárias porque os percentuais de ausentes ou insuficientes são altos em metade dos documentos (GRÁFICO 1B).

As variáveis do subsistema Governança foram as mais ausentes dos documentos (GRÁFICO 1C), 
seguida das variáveis do subsistema Atores (GRÁFICO 1D). Lembrando que esses subsistemas refletem a capacidade de governança e gestão das demandas para sustentabilidade do recurso, assim como participação dos atores no processo.

\section{DISCUSSÃO}

Para Duarte et al. (2017), era de se esperar que os estudos apresentados como requisito para emissão de licenças ambientais apresentassem padrão de alta qualidade para responder às demandas dos atores locais envolvidos, governo e Organizações Não Governamentais. No entanto, ao revisar trabalhos dessa natureza os autores encontraram sérias deficiências no processo de Avaliação de Impacto Ambiental, evidenciando uma lacuna entre planejamento e execução. Mesmo assim, recentemente tem aparecido ataques políticos à obrigatoriedade do licenciamento ambiental para empreendimentos hidrelétricos, materializados na Proposta de Emenda Constitucional 65 de 2012 (PEC 65/2012), que mostram que os investidores e instâncias do Estado encontram nesse sistema um entrave para as obras. A legislação ambiental brasileira é percebida, por muitos investidores, como entrave porque ela encarece, alonga e cria instabilidade socioeconômica ao longo do processo de licenciamento (HOCHSTETLER, 2011).

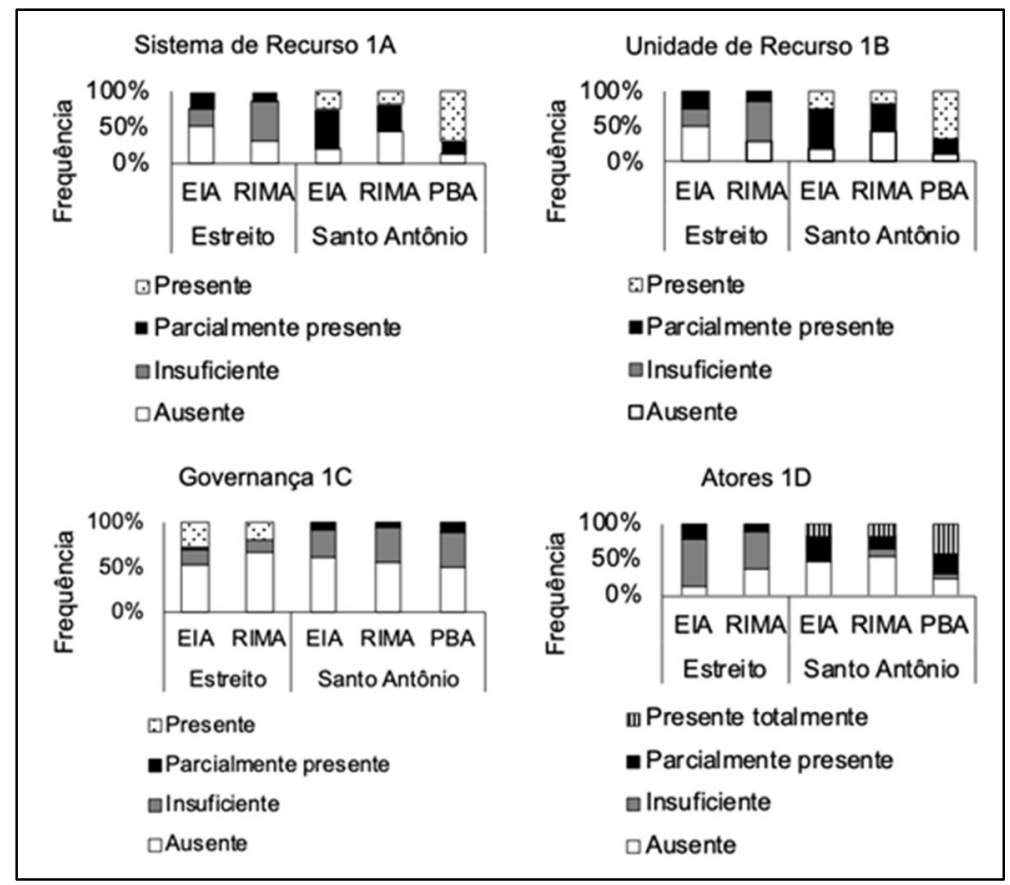

Gráfico 1: Comportamento das variáveis do subsistema 1A) Sistema de Recurso (SR), 1B) Unidade de Recurso (UR), 1C) Governança (G) e 1D) Atores (A) presentes nos documentos de licenciamento ambiental das hidrelétricas de Estreito e Santo Antônio, segundo as categorias ausente, insuficiente, presente parcialmente e presente.

Na contramão disso, pesquisas científicas como está, indicam a necessidade do aprimoramento do sistema de licenciamento e da atualização do arcabouço teórico que os orienta (AGOSTINHO et al., 2004; DORIA et al., 2017; FEARNSIDE, 2014; WINEMILLER et al., 2016). As pesquisas de Ostrom e seguidores (BASURTO et al., 2013; BINDER et al., 2013; COX, 2014; OVIEDO et al., 2016; TRIMBLE et al., 2015), permitem afirmar também que a sustentabilidade de recursos de uso comum está ligada a possibilidade de sua gestão compartilhada (WALL, 2014). Os enunciados de Hardin de que as opções, em termos de gestão, para aqueles 
recursos eram a privatização ou a estatização foram sendo derrubados à luz dos mais de cem casos bemsucedidos de gestão coletiva de recursos como água, peixes, florestas e pastagens estudados por Ostrom (1990). Esses recursos de uso comum - se geridos de forma coletiva (pela população usuária do recurso) e sob certas condições favoráveis à ação coletiva, como a definição de fronteiras, a prestação de contas, a importância do recurso para o usuário entre os outros princípios institucionais (OSTROM, 1990) impediriam a sua depleção e garantiriam a sua manutenção para as gerações futuras.

Ainda segundo Wall (2014), o mundo passa atualmente por uma onda de fechamento (enclosers) dos comuns (por meio de privatização, principalmente) sem precedentes na história. Sendo ele um estudioso dos "fechamentos" dos comuns na Inglaterra no século XVII, ele traça uma linha entre a expansão da economia de mercado e a privatização dos recursos de uso comuns (WALL, 2014). Nesse sentido, a sustentabilidade dos comuns no mundo contemporâneo passa pela possibilidade de resistir à expansão da economia de mercado sobre os recursos de uso comum. As referidas críticas ao sistema de licenciamento ambiental parecem estar ligadas a necessidade de barateamento dos custos dos empreendimentos e se orientam, assim, pela perspectiva de que a mercantilização dos recursos é a solução, cabendo ao Estado livrar os investidores dos entraves.

Como afirmam os autores Agrawal (2001) e Wall (2014), concorda-se que a falência de muitos destes sistemas de recursos está ligada não apenas aos problemas de gestão e as suas múltiplas relações. As pressões externas do mercado e do estado têm contribuindo para a depleção de recursos de uso comum geridos, até a chegada desses atores externos, coletivamente. Nesse sentido, outras questões como o nível de impacto antrópico sofrido, a diversidade biológica entre outros fatores, podem contribuir para uma boa ou má gestão, da mesma maneira que as variáveis observadas no arcabouço do SSE. Com essa crítica em mente, foi preciso fortalecer as características sociais, econômicas e políticas na adaptação proposta para o arcabouço de SSE, de modo a adequá-lo à realidade Amazônica atual, que enfrenta um intenso processo de expansão da economia de mercado (CASTRO, 2012; MORAN, 2016). Existem evidências claras de que os espaços como 'os rios', que até recentemente eram poucos afetados pela modelo de produção capitalista orientado para a privatização dos recursos de uso comum como a água e pesca, estão passando por processos de fechamento e semiprivatização. Por outro lado, como afirma Linebaugh (2014) para uma visão realmente sistemática dos commons, precisamos reconhecer que

The commons is not only "common-pool resources" no is the commons purely "the people." In other words it is not a thing but a relationship [...] the commoner was the person who commoned in such lands [...] In this struggle our landscapes were formed even our human "nature" as well as nature herself.

Assim, o arcabouço de Ostrom $(2009 ; 2012)$ torna-se muito útil para a análise dos arranjos de regime de propriedade e de gestão que emergiram na atualidade, em que o Estado e as companhias privadas fazem a gestão compartilhada dos recursos. Isso se dá, por exemplo, quando o Estado concede a exploração de recursos comuns para companhias privadas, como é o caso das parcerias público-privadas no setor elétrico brasileiro, ou para territórios em disputa entre o capital privado, o Estado e as populações locais, no caso dos rios amazônicos. Contudo, os novos arranjos parecem potencializar a força dos seus enunciados, visto que 
essas novas formas de institucionalização da gestão dos recursos são de fato a privatização da exploração e a estatização do regime de propriedade. E, como esse novo arranjo não é nem a estatização pura nem na privatização pura, vários atores, como os pescadores, são afastados da discussão sobre os recursos e o seu uso (DORIA et al., 2017). Ou são, como os pescadores artesanais, afetados diretamente (DORIA et al., 2017).

Nesse sentido, é preciso reconhecer que os commons são, como defende Wall (2015), cultura e organização política. No caso da Amazônia, existem as especificidades dos recursos, motivadas pelas características próprias deste ecossistema, como as comunidades foram construindo esse ambiente de forma dialógica ao longo do tempo, modificando-o e se modificando nesse processo (HECKENBERGER, 2004). Assim, as comunidades ou os comunais, como afirma Linebaugh (2014), possuem um conhecimento sofisticado sobre o sistema ecológico elaborado na sua relação de longa data com os recursos que precisam ser conhecidos e considerados (ESCOBAR, 2008).

Ainda como pontuam Dardot et al. (2015), os comuns são um princípio político de gestão dos recursos que não apenas existe em comunidades tradicionais, mas tem sido "la reinvidicacion [...] em la luchas sociales y culturales contra el orden capitalista y el Estado empresarial" (DARDOT et al., 2015). Isso pode ser visto, por exemplo, nos discursos dos movimentos sociais amazônicos como o Movimento dos Atingidos por Barragens (MAB) quando afirmam 'água e energia não são mercadorias'. O reconhecimento dos commons como um sistema de propriedade e uso baseado em um tipo de pensamento político, ajuda a reconhecer o projeto político dos grupos tradicionais e a empoderá-los, de forma a diminuir a falta de equidade na tomada de decisão sobre os destinos dos rios, peixes e outros recursos Amazônicos de uso comum.

Além das características do subsistema social amazônico, no qual se enfatizou o conhecimento tradicional sobre os recursos e o contexto histórico de avanço do mercado sobre os recursos de uso comum na região, foi preciso dar nova ênfase às características do subsistema ecológico. O regime hidrológico e as características da ictiofauna amazônica, principalmente aquela de valor comercial, levaram à necessidade de pensar os atributos desse subsistema de forma diferenciada. Um exemplo são os grandes bagres migradores, que tem como nicho praticamente toda a extensão das bacias fluviais onde vivem - e às vezes interconectamse a bacias próximas (DUPONCHELLE et al., 2016; HEGG et al., 2015). Assim, nesta análise, foi preciso considerar os atributos específicos do ambiente aquático tropical amazônico no esforço de teorizar os atributos do sistema ao adaptar para ele o arcabouço proposto por Ostrom.

\section{CONCLUSÕES}

Ao identificar uma série de variáveis-chave que permitam a descrição do Sistema Pesqueiro Amazônico (SSESPA) de forma integrativa, em ambientes afetados por grandes barragens hidrelétricas, o presente trabalho mostra a pertinência da adaptação do arcabouço de Sistemas Socioecológicos (SSE) de Ostrom (2009) e Basurto et al. (2013) para embasar tomadas de decisão orientadas para a sustentabilidade deste sistema no contexto estudado. Contudo, no que diz respeito às múltiplas relações entre os diversos 
atributos, variáveis e escalas do SPA, o arcabouço desenvolvido demonstra também as lacunas de informação existentes nos estudos de impacto ambiental que podem comprometer a implementação de medidas compensatórias ou mitigadoras dos impactos. Isso porque os documentos para a emissão de licenças ambientais de hidrelétricas têm conseguido produzir dados mínimos sobre a configuração desses sistemas.

Os modelos vigentes nos documentos de Licenciamento ambiental como EIAs, RIMAs e PBAs e que acabam por orientar a gestão da pesca em hidrelétricas na Amazônia parece inadequado e impróprio para a promoção da sustentabilidade dos recursos pesqueiros. Mesmo assim, os modelos de EIAs, RIMAs e PBAs tem sido replicados e até considerados como longos, dispendiosos e passíveis de serem flexibilizados. Combatendo este argumento, o presente trabalho reafirma a necessidade de maior integração, consideração dos conhecimentos locais e aprofundamento dos estudos sobre os recursos pesqueiros de modo a embasar tomadas de decisão mais efetivas. Para tal, propõe um guia de cinco passos para a aplicação do arcabouço de Sistemas Socioecológicos para o Sistema Pesqueiro Amazônico (SSE/SSESPA). O passo I, do guia, refere-se ao acesso à informação. Nessa primeira etapa, representantes dos atores envolvidos no processo (pescadores, Organizações governamentais e não governamentais, empresa, academia, etc.) discutem o checklist de variáveis aqui proposto. A partir dessa discussão, adapta-se o SSE/SPA para ser aplicado em EIAs, RIMAs e PBAs, de forma experimental (passo II). Após a aplicação, faz-se uma avaliação do cumprimento do checklist e da viabilidade de sua aplicação (passo III). A partir disso, fazem-se reajustes necessários (passo IV), reeditando o SSE /SPA e o adotando no processo de licenciamento ambiental, no que se refere ao impacto das barragens na pesca (passo V). As ações de monitoramento, análise e aprendizado devem se dar de forma contínua ao longo de todo o processo de implementação. Considerando o nível de aprofundamento requerido para estudos que avaliem o real impacto das barragens na pesca e nos peixes, análises dessa natureza se efetivam nos médio e longo prazos.

A necessidade da adaptação dos atributos e variáveis à realidade amazônicas, bem como lidar com as críticas ao arcabouço de Ostrom (2009), mostra, por um lado, a necessidade de se continuar estudando os impactos sobre os sistemas socioecológicos amazônicos e, por outro, indicam a pertinência da adaptação do arcabouço para os sistemas estudados na condução da realização dos estudos de Licenciamento Ambiental no que diz respeito ao sistema da pesca artesanal. As evidências, conforme apontam os resultados, de que os estudos não alcançam o conhecimento aprofundamento do sistema está colocado pelo fato de que nos EIAs, RIMAs e PBAs estudados, os atributos e variáveis chaves do sistema estão ausentes ou parcialmente presentes. Mesmo sendo uma proposta que requer tempo para a análise, reforçamos a necessidade da tomada de medidas adequadas e urgentes para evitar o colapso dos recursos pesqueiros nesses ambientes, impactando ainda mais a vida de milhares de família de baixa renda, que dependem da pesca para sua sobrevivência.

\section{REFERÊNCIAS}


AGOSTINHO, A. A.; GOMES, L. C.; LATINI, J. D.. Fish diversity in the upper Paraná River basin: Habitats, fisheries, management and conservation. Aquatic Ecosystem Health and Management, v.10, n.2, p.174-86, 2007.

AGOSTINHO, A. A.; GOMES, L. C.; LATINI, J. D.. Fish assemblages in Neotropical reservoirs: Colonization patterns, impacts and management. Fisheries Research, v.173, p.26-36, 2016.

AGRAWAL, A.. Common Property Institutions and Sustainable Governance of Resources. World Development, v.29, n.10, p.1649-72, 2001.

BASURTO, X.; GELCICH, S.; OSTROM, E.. The social-ecological system framework as a knowledge classificatory system for benthic small-scale fisheries. Global Environmental Change, v.23, n.6, p.1366-380, 2013.

BERKES, F.. Restoring Unity: The Concept of Marine SocialEcological Systems. In: WORLD Fisheries: A Social-Ecological Analysis. Blackwell, 2011. p.9-28.

BHAUMIK, U.; MUKHOPADHYAY, M. K.; SHRIVASTAVA, N. P.; SHARMA, A. P.; SINGH, S. N.. A case study of the Narmada River system in India with particular reference to the impact of dams on its ecology and fisheries. Aquatic Ecosystem Health and Management, v.20, n.1-2, p.151-9, 2017. DOI: https://doi.org/10.1080/14634988.2017.1288529

BINDER, C. R.; HINKEL, J.; BOTS, P. W. G.; PAHL-WOST, C. Comparison of frameworks for analyzing social-ecological systems. Ecology and Society, v.18, n.4, 2013.

BUSCHBACHER, R.. A Teoria da Resiliência e os Sistemas Socioecológicos: como se preparar para um futuro imprevisível.? Boletim Regional, Urbano e Ambiental, v.9, p.11-24, 2014.

CASTRO, E.. Expansão da fronteira, megaprojetos de infraestrutura e integração sul-americana. Caderno CRH, v.25, n.64, p.45-61, 2012.

CESTE. Consórcio Estreito Energia. Usina Hidrelétrica Estreito. 2018.

COOPER, A. R.; INFANTE, D.; WEHRLY, K. E.; WANG, L.. Identifying indicators and quantifying large-scale effects of dams on fishes. Ecological Indicators, v.61, p.646-657, 2016.

COX, M.. Applying a Social-Ecological System Framework to the Study of the Taos Valley Irrigation System. Human Ecology, v.42, p.311-24, 2014.

DARDOT, P.; LAVAL, C.. Propriedade, apropriação social e instituição do comum. Tempo Social, v.27, n.1, p.261-73, 2015.

DORIA, C. R. C.; RUFFINO, M. L.; HIJAZI, N. C.; CRUZ, R. L.. A pesca comercial na bacia do rio Madeira no estado de Rondônia, Amazônia brasileira. Acta Amazônica, v.42, n.1, p.29-40, 2012. DOI: https://doi.org/10.1590/S0044$\underline{59672012000100004}$

DORIA, C. R. C.; LIMA, M. A. L.. Rio Madeira Seus Peixes Sua Pesca. Porto Velho: EDUFRO, 2017.
DORIA, C. R. C.; ATHAYDE, S.; MARQUES, E.; LIMA, M. A. L.. The invisibility of fisheries in the process of hydropower development across the Amazon. Ambio, v.47, n.4, p.45365, 2017. DOI: http://doi.org/10.1007/s13280-017-0994-7

DUARTE, C. G.; DIBO, A. P. A.; SÁNCHEZ, L. E.. What does the academic research say about impact assessment and environmental licensing in Brazil?. Ambiente e Sociedade, v.20, n.1, p.261-92, 2017.

DUPONCHELLE, F.; POUILLY, M.; PÉCHEYRAN, C.; HAUSER, M.; RENNO, J.-F.; PANFILI, J.; DARNAUDE, A. M.; VASQUEZ, A. G.; CARVAJAL-VALLEJOS, F. M.; DAVILA, C. G.; DORIA, C.; BERAIL, S.; DONARD, A.; SONDAG, F.; SANTOS, R. V.; NÚÑEZRODRIGUEZ, J.; POINT, D.; LABONNE, M.; BARAS, E.. TransAmazonian natal homing in giant catfish. Journal of Applied Ecology, v.53, n.5, p.1511-1520, 2016.

ESCOBAR, A.. Territories of difference: Place, Movements, Life, Redes. Durham: Duke University Press, 2008.

FEARNSIDE, P. M.. Impacts of Brazil's Madeira River Dams: Unlearned lessons for hydroelectric development in Amazonia. Environmental Science \& Policy Policy, v.38, p.164-72, 2014.

HARDIN, G.. The Tragedy of the Commons. Science, v.162, n.3.859, p.1243-48, 1968.

HECKENBERGER, M. J.. The ecology of power: Culture, place and personhood in the Southern Amazon, AD 1000-2000. Routledge, 2004.

HEGG, J. C.; GIARRIZZO, T.; KENNEDY, B. P.. Diverse early lifehistory strategies in migratory Amazonian catfish: Implications for conservation and management. PLoS ONE, v.10, n.7, p.e0129697, 2015.

HOCHSTETLER, K.. The Politics of Environmental Licensing: Energy Projects of the Past and Future in Brazil. Studies in Comparative International Development, v.46, n.4, p.34971, 2011.

KALIKOSKI, D. C.; SEIXAS, C. S.; ALMUDI, T.. Gestão compartilhada e comunitária da pesca no Brasil: Avanços e desafios. Ambiente e Sociedade, v.12, n.1, p.151-172, 2009.

LINEBAUGH, P.. Stop, Thief! The commons, enclosures and resistance. PM Press, 2014.

LITTLE, P. E.. Territórios sociais e povos tradicionais no Brasil: por uma antropologia da territorialidade. Anuário Antropológico, p.251-90, 2002.

MORAN, E. F.. Roads and dams: Infrastructure-driven transformations in the Brazilian Amazon. Ambiente e Sociedade, v.19, n.2, p.207-20, 2016.

OLIVEIRA, J. G. C.; SILVA, L. P. S.; MALHADO, A. C. M.; BATISTA, V. S.; FABRÉ, N. N.; LADLE, R. J.. Artisanal fisheries research: A need for globalization?. PLoS ONE, v.11, n.3, p.e0150689, 2016. DOI: https://doi.org/10.1371/journal.pone.0150689

OLIVER-SMITH, A.. Defying displacement: Grassroots 
resistance and the critique of development. Austin:

University of Texas Press, 2010.

OSTROM, E.. Governing the commons: The evolution of institutions for collective action. Cambridge: Cambridge University Press, 1990.

OSTROM, E.. A general framework for analyzing sustainability of social-ecological systems. Science, v.325, p.419-22, 2009.

OSTROM, E.. The future of the commons: Beyond Market Failure and Government Regulation. The Institute of Economic Affairs, 2012.

OVIEDO, A. F. P.; BURSZTYN, M.. The Fortune of the Commons: Participatory Evaluation of Small-Scale Fisheries in the Brazilian Amazon. Environmental Management, v.57, n.5, p.1009-023, 2016.

POTEETE, A. R.; JANSSEN, M. A.; OSTROM, E.. Working together: Collective action, the commons, and multiple methods in practice. 2010.
SÁNCHEZ, L. E.. Avaliação de impacto ambiental: conceitos e métodos. 2013.

SANTOS, G. M.; SANTOS, A. C. M.. Sustentabilidade da pesca na Amazônia. Estudos Avançados, v.19, n.54, p.165-82, 2005.

SEVÁ FILHO, A. O.. Estranhas catedrais: notas sobre o capital hidrelétrico, a natureza e a sociedade. Ciência e Cultura, v.60, n.3, p.44-50, 2008.

TRIMBLE, M.; BERKES, F.. Towards adaptive co-management of small-scale fisheries in Uruguay and Brazil: lessons from using Ostrom's design principles. Maritime Studies, v.14, n.14, p.2-20, 2015.

WALL, D.. The Commons in History. Culture, Conflict, and Ecology. The MIT Press, 2014.

WINEMILLER, K. O. et al. Balancing hydropower and biodiversity in the Amazon, Congo, and Mekong. Science, v.351, n.6269, p.128-9, 2016. DOI: http://doi.org/10.1126/science.aac7082

A CBPC - Companhia Brasileira de Produção Científica (CNPJ: 11.221.422/0001-03) detém os direitos materiais desta publicação. Os direitos referem-se à publicação do trabalho em qualquer parte do mundo, incluindo os direitos às renovações, expansões e disseminaçães da contribuição, bem como outros direitos subsidiários. Todos os trabalhos publicados eletronicamente poderão posteriormente ser publicados em coletâneas impressas sob coordenação da Sustenere Publishing, da Companhia Brasileira de Produção Científica e seus parceiros autorizados. Os (as) autores (as) preservam os direitos autorais, mas não têm permissão para a publicação da contribuição em outro meio, impresso ou digital, em português ou em tradução. 